\title{
Multi-accelerator extension in OpenMP based on PGAS model
}

\author{
Masahiro Nakao \\ RIKEN Center for Computational \\ Science \\ Hyogo, Japan
}

\author{
Hitoshi Murai \\ RIKEN Center for Computational \\ Science \\ Hyogo, Japan
}

\author{
Mitsuhisa Sato \\ RIKEN Center for Computational \\ Science \\ Hyogo, Japan
}

\begin{abstract}
Many systems used in HPC field have multiple accelerators on a single compute node. However, programming for multiple accelerators is more difficult than that for a single accelerator. Therefore, in this paper, we propose an OpenMP extension that allows easy programming for multiple accelerators. We extend existing OpenMP syntax to create Partitioned Global Address Space (PGAS) on separated memories of several accelerators. The feature enables users to perform programming to use multiple accelerators in ease. In performance evaluation, we implement the STREAM Triad and the HIMENO benchmarks using the proposed OpenMP extension. As a result of evaluating the performance on a compute node equipped with up to four GPUs, we confirm that the proposed OpenMP extension demonstrates sufficient performance.
\end{abstract}

\section{CCS CONCEPTS}

- Software and its engineering $\rightarrow$ Parallel programming languages;

\section{KEYWORDS}

OpenMP, accelerator, partitioned global address space, parallel programming

\section{ACM Reference Format:}

Masahiro Nakao, Hitoshi Murai, and Mitsuhisa Sato. 2019. Multi-accelerator extension in OpenMP based on PGAS model. In International Conference on High Performance Computing in Asia-Pacific Region (HPC Asia 2019), January 14-16, 2019, Guangzhou, China. ACM, New York, NY, USA, 8 pages. https://doi.org/10.1145/3293320.3293324

\section{INTRODUCTION}

Accelerators with excellent power performance ratio and memory bandwidth are widely used as computing resources. Many of the systems ranked on the latest Top500 list (http://www.top500.org) and the Green500 list (http://www.green500.org) in June 2018 are equipped with accelerators such as NVIDIA GPU, Intel Xeon Phi, and PEZY company PEZY-SC2. A single compute node in such systems often has multiple accelerators to improve the power performance ratio further. For example, "Summit"[15] in Oak Ridge National Laboratory, which is ranked No. 1 in Top500 list above, has six NVIDIA Tesla V100 GPUs per compute node.

Permission to make digital or hard copies of part or all of this work for personal or classroom use is granted without fee provided that copies are not made or distributed for profit or commercial advantage and that copies bear this notice and the full citation on the first page. Copyrights for third-party components of this work must be honored. For all other uses, contact the owner/author(s).

HPC Asia 2019, January 14-16, 2019, Guangzhou, China

(c) 2019 Copyright held by the owner/author(s)

ACM ISBN 978-1-4503-6632-8/19/01.

https://doi.org/10.1145/3293320.3293324
As accelerator programming languages, CUDA[14], OpenCL[24], OpenMP[18], and OpenACC [17] are often used. Specifically, OpenMP and OpenACC are directive-based languages, so you can easily develop a parallel code for an accelerator from a sequential code for a host. In addition, since OpenMP and OpenACC do not depend on a specific architecture, they also have an advantage of high portability.

However, as an issue of the accelerator programming languages, it is difficult to deal with multiple accelerators. The reason is that since the memory spaces of the accelerators are physically and logically separated, it is necessary for users to divide data and tasks to each accelerator manually. An issue also exists in distributed memory systems such as PC clusters. Although MPI is widely used for the programming environment in such systems, programming is generally difficult since it is necessary to divide data and tasks to each compute node. To solve this issue, Partitioned Global Address Space (PGAS) has been proposed as a programming model aiming at achieving both high productivity and high performance[1, 2, 20, 22]. PGAS provides a global memory space composes of local memories in execution units, and each execution unit can freely access the global memory space. Moreover, since an affinity between the local memory and each execution unit is presented, its feature enables users to do programming with data locality awareness.

In this paper, we propose an extension that can handle PGAS for OpenMP to deal with multiple accelerators in ease. Some of the OpenMP extension refer to XcalableMP (XMP)[11,27] which is a directive-based PGAS language for distributed memory systems.

The remainder of this paper is structured as follows. Section 2 presents related works. Section 3 explains an example of how to deal with multiple accelerators using existing OpenMP syntax. Section 4 proposes new OpenMP extension for multiple accelerators. Section 5 evaluates its performance. Section 6 concludes this paper and discusses areas for future research.

\section{RELATED WORK}

Although existing accelerator programming languages support only a single accelerator, there are several methods to deal with multiple accelerators by using them together with parallel programming languages for hosts. For example, (1) spawn multiple threads using OpenMP on host, and each thread deals with one accelerator [6, 8, 25], (2) spawn multiple processes using MPI on host, each process deals with one accelerator[21,25]. The method (1) covers only a single compute node, whereas the method (2) can also cover on a distributed memory system equipped with accelerators (accelerated cluster). However, the method (2) requires that the data used by the host also be divided to each process as well as the data used by the accelerator, so the programming complexity of the method (2) may be higher than that of the method (1). 
Although there is no research to deal with multiple accelerators using OpenMP, several researches use OpenACC. Xu et al.[28] propose an OpenACC extension to support multiple accelerators. Although the OpenACC extension supports communication between accelerators, it is necessary to divide data and tasks manually. Komoda et al.[23] propose another OpenACC extension which supports to divide data and tasks into multiple accelerators. Furthermore, its compiler system has a mechanism to keep data consistency on the accelerator memory automatically. However, the OpenACC extension can be used only just before the loop statement. Therefore, the OpenACC extension cannot upload data in an arbitrary place. Matsumura et al.[12] develop an OpenACC compiler system to generate an OpenACC code for multiple accelerators from an OpenACC code for a single accelerator automatically. However, there are some limitations. For example, a loop statement that can be divided is composed only of affine access. Also, since it is necessary to transfer all data on the host used for calculation to each accelerator, the memory capacity that can be used by multiple accelerators is the same as only one accelerator. Nakao et el.[10, 26] develop an XcalableACC directive-based language for accelerated clusters. XACC is a new language in which XMP and OpenACC are vertically integrated, using XMP for host side programming and OpenACC for accelerator side programming. XACC also provides directives to transfer data between accelerators and to divide data and tasks into multiple accelerators. Even for a single compute node, XACC supports functions to deal with multiple accelerators, but it is necessary to use XMP syntax for host programming as well as OpenACC for accelerator programming.

\section{ACCELERATOR PROGRAMMING WITH OPENMP}

\subsection{Overview}

The OpenMP specification 1.0 supported only Fortran, which was released in October 1997, and next specification also supported C and $\mathrm{C}++$, which was released in October 1998. Although target systems of before the OpenMP specification 4.0 were shared memory systems, the OpenMP specifications 4.0 also supported accelerators released in July 2013. The latest specification at present is OpenMP 4.5 released in November 2015. In this paper, OpenMP 4.0 and later is called "OpenMP 4.x."

A compiler list compatible with OpenMP is described on the OpenMP official site[19]. Among them, OpenMP compilers that are open source and deal with accelerators are Heterogeneous OpenMP (for NVIDIA GPU)[9], Clang (for NVIDIA GPU)[3], and GNU GCC (for Intel MIC)[4].

\subsection{Comparison with OpenACC}

OpenACC is a directive-based accelerator programming language like OpenMP 4.x. As with OpenMP, OpenACC also supports Fortran, $\mathrm{C}$, and $\mathrm{C}++$. OpenMP has been expanded to support accelerators from shared memory systems, whereas OpenACC supports accelerators from the beginning. Thus, the OpenACC specification is less complicated than the OpenMP specification. In addition, the first specification of OpenACC was released in November 2011, and it is earlier than the release of OpenMP 4.0. For these reasons,

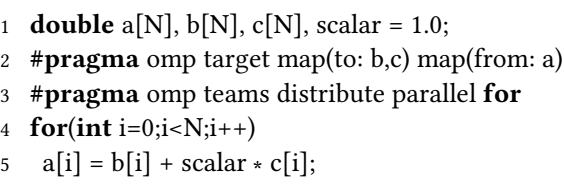

Figure 1: STREAM Triad using OpenMP for a single accelerator

as mentioned in Section 2, researches for an accelerator programming is more active on OpenACC than OpenMP at present.

The syntaxes and programming models of OpenMP 4.x and OpenACC for accelerators are very similar. Both OpenMP 4.x and OpenACC provide directives to transfer data between host and accelerator and to execute a loop statement on the accelerator. As for the difference with the data management policy, OpenACC can automatically generate data-transfer between host and accelerator, OpenMP requires users to describe it explicitly. Also, as a difference in the function, for example, the latest OpenACC 2.6 supports a deep copy of data structures with pointers, whereas OpenMP 4.x does not support it. In contrast, OpenMP 4.x provides the device clause to specify an accelerator, whereas OpenACC 2.6 does not provide such a clause. However, as for the function, since each specification affects each other, we consider that the difference will become small in the future, or it will be unified into one.

The reason we focus on OpenMP in this paper is that OpenMP has more various functions for a host (CPU thread) than OpenACC. To exploit all computing resources of a compute node equipped with accelerators, it is indispensable to use a host as well as accelerators. For example, when OpenACC is used for accelerator programming, host-side computation uses OpenMP, so two kinds of programming languages are mixed in one code. On the other hand, using only OpenMP, programming for heterogeneous computing resources can be done in one language, so the educational cost can be kept low.

\subsection{Example of Single Accelerator Programming}

Fig. 1 shows an example of accelerator programming using OpenMP. This example is a simplified version of the STREAM Triad benchmark in HPC Challenge Benchmark Suite[7]. In line 2, the target directive indicates that it is a kernel that operates on an accelerator within its range (lines 2 to 5 ). The map clause on the same line instructs the transfer of data between host and accelerator. It transfers the arrays $b[]$ and $c[]$ from host to accelerator before the kernel execution, and the array $a[]$ from accelerator to host after the kernel execution. In line 3, the teams distribute parallel for directive is a shortcut to execute following multiple actions. (1) The teams construct creates a league of thread teams. The team is a coarse-grain parallel level unit containing multiple threads. (2) The distribute construct instructs a following loop statement to be divided by the master thread of each team. (3) The parallel for construct executes a following loop statement by the multiple threads of the teams in parallel. 


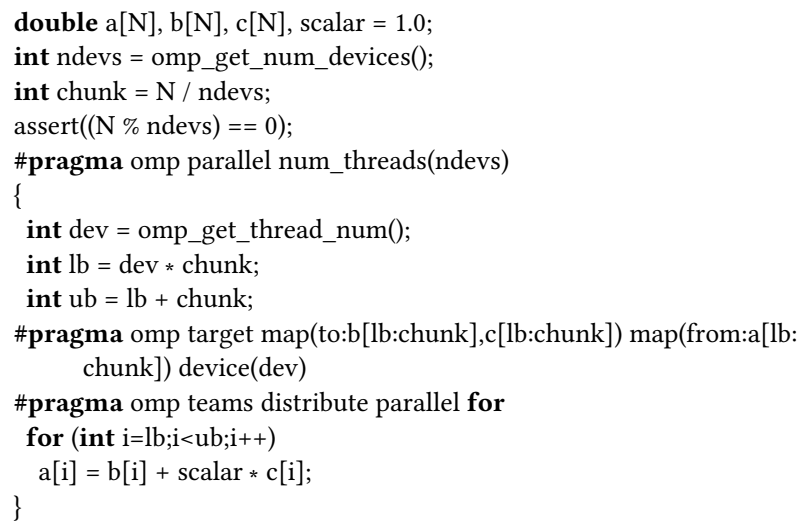

Figure 2: STREAM Triad using OpenMP for multiple accelerators

\subsection{Example of Multiple Accelerators Programming}

Fig. 2 shows an example of parallelizing the STREAM Triad benchmark shown in Fig. 1 for multiple accelerators. In line 2, the function omp_get_num_devices() returns the number of accelerators on the host. In line 3, a variable chunk which is the number of iterations and the data size for one accelerator is calculated. In this example, it is assumed that the total iteration number $N$ is divisible by the number of accelerators ndevs, but it is also necessary to consider the case where it is not divisible in general. In line 5 , to make each host thread deal with one accelerator, the parallel directive spawns host threads the number of which is the same as the number of accelerators. In line 7 , the function omp_get_thread_num() returns a host thread number. In lines $8-9$, the range of iterations executed on each accelerator is calculated. In line 10, the target directive indicates that the accelerator specified in the device clause executes its range (lines 10 to 13). In the arrays specified in the map clause on the same line, the array section is used to map only a certain range of the array. In array section, the number to the left of the colon in the square bracket of the array is the start transfer element, and the number to the right of the colon is the number of transfer elements. In lines 11-13, each accelerator calculates the responsible range.

As can be seen from this example, when using multiple accelerators, it is necessary to divide data and tasks to each accelerator explicitly. Also, in the case of an application that does not appear in this example but requires communication between accelerators, it is necessary to generate communication using the function omp_target_memcpy() provided by OpenMP for the divided data on each accelerator. These procedures are similar to programming using MPI in a distributed memory system and are very complicated.

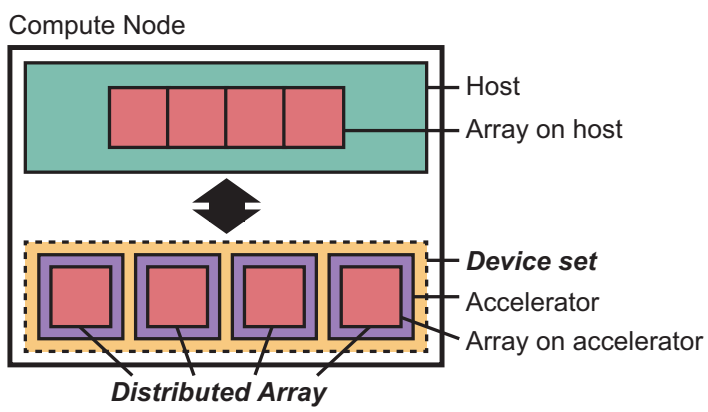

Figure 3: Concept of OpenMP extension

\section{OPENMP EXTENSION FOR MULTIPLE ACCELERATORS}

\subsection{Design}

In this section, we propose the OpenMP extension for using multiple accelerators easily. Specifically, we propose OpenMP directives to divide tasks and data and perform them on multiple accelerators. Since XMP directives can divide tasks and data and perform them on distributed memory systems, this is very similar to using multiple accelerators. Thus, we propose the OpenMP extension based on XMP directives.

Fig. 3 shows a concept of the OpenMP extension, where new terms device set and distributed array are declared. The device set is a set of accelerators on a single host. The OpenMP extension can deal with multiple accelerators like a single accelerator by describing orders to the device set. The distributed array is a virtual array divided on the device set. One array on host memory is divided and upload onto multiple accelerator memories as a distributed array. The OpenMP extension can perform PGAS-like operations for the distributed array.

The OpenMP extension provides following functions.

- Declaration of device set

- Declaration of distributed array

- Communication between host and device set

- Communication in device set

\subsection{Specification}

This section describes the OpenMP extension using some examples. The contents of the extension are as follows.

- To declare device set, an existing device clause is extended to use array section

- To divide data and tasks, a new layout directive is added. The layout directive appears with existing map clause, declare target directive, distribute directives.

- To develop stencil applications easily, new shadow clause and target reflect directive are added. The shadow clause appears with layout clause.

- To communicate in device set, new target gmove, target bcast, and target reduction directives are added.

Fig. 4 shows an example of parallelizing the STREAM Triad benchmark shown in Fig. 1 using the OpenMP extension for multiple accelerators. The operation of Fig. 4 is almost the same as 


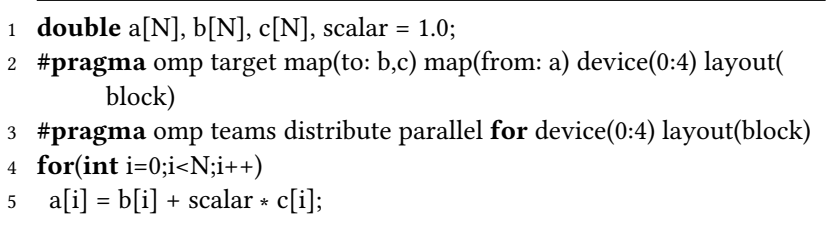

Figure 4: Example of STREAM Triad using extended OpenMP

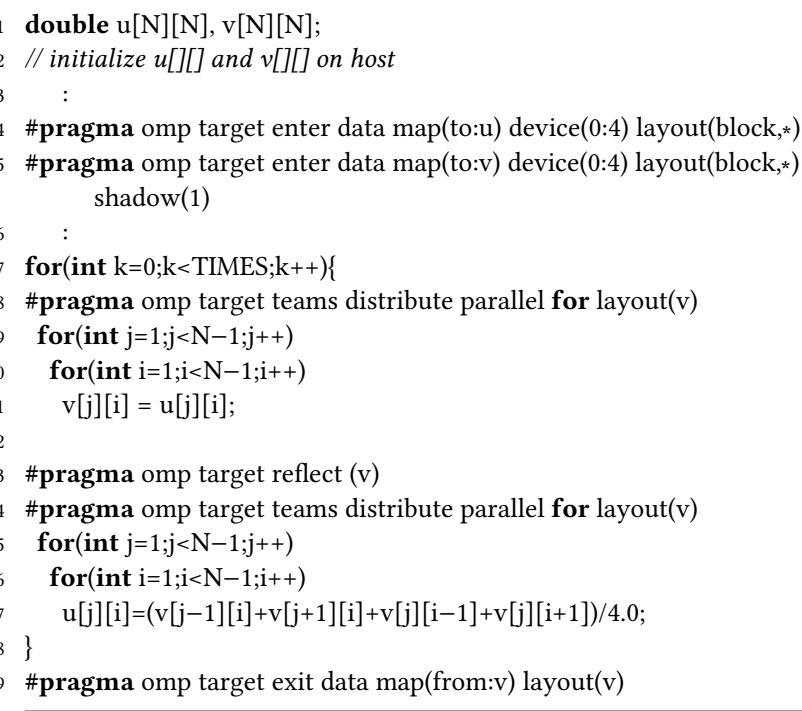

Figure 5: Example of Laplace equation using extended OpenMP

Fig. 2, and Fig. 4 is only added device clause and layout clause to Fig 1 . In lines $2-3$, the device clauses indicate that a device set composed of four devices device(0) to device(3) is used. When you specify an asterisk like device $\left({ }^{*}\right)$, it means all the devices on a host. In line 2, the layout clause divides the array $a[], b[]$, and $c[]$ in a block manner and maps them to each device. This block manner supports a case where the size of the array is not divisible by the number of devices. For example, device $(0)$ has elements of a[0:ceiling(N/4)] (ceiling() is a ceiling function), and the last device device(3) has elements of $a\left[3^{*}\right.$ ceiling(N/4):N-3*ceiling(N/4)]. If more than one accelerator is specified in the device clause and there is no layout clause, an array specified in the map clause is duplicated and stored in each accelerator. In line 3, the layout clause indicates that the iteration of the following loop statement is divided in a block manner. To support a case of the size of the array does not match the iteration of the loop statement, the layout clause can also specify the array name as in \#pragma omp teams distribute parallel for layout(a). In this case, the iteration of the loop statement is divided according to the division of the specified array. Furthermore, when an array name is specified, a compiler system can acquire the device set information from the array, so that the device clause can be omitted.

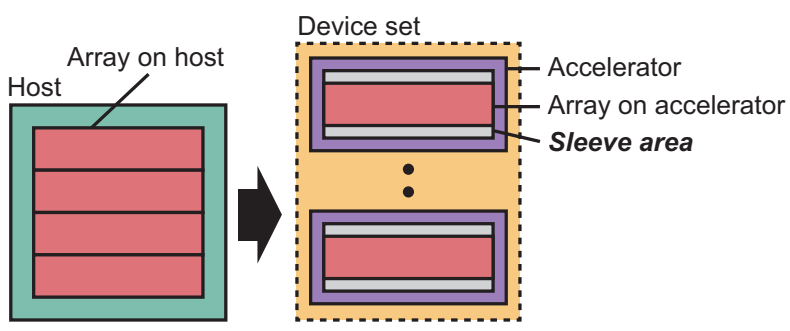

Figure 6: Concept of shadow clause

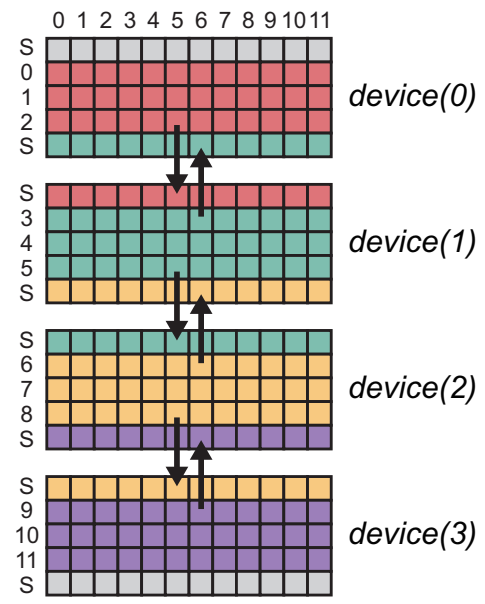

Figure 7: Concept of target reflect directive ("S" means sleeve area)

Next, Fig. 5 shows how to divide a multi-dimensional array to develop a stencil application on the two-dimensional Laplace equation. In lines 4-5, the target enter data directive divides the first dimension of arrays $u[][]$ and $v[][]$ and map them to the device set. The dimension with an asterisk in the layout clause means that its dimension is not divided. When dividing multiple dimensions, you declare a multi-dimensional device set and map a multi-dimensional array to it like \#pragma omp target enter data map(to:u) device(0:2,0:2) layout(block,block). In line 5, the shadow clause adds a sleeve area of width " 1 " required for stencil calculation to the upper and lower bounds of the array $v[][]$. Fig. 6 shows a concept of the shadow clause in this example. In lines $8-11$ and $14-17$, only the outermost loop is divided in a block manner, and the four accelerators execute the divided loop statement in parallel. In line 13, the target reflect directive generates communication in accelerators to update the sleeve area of the distributed array. Fig. 7 shows a concept of the target reflect directive in this example where the size of the array is 12 $(N=12)$. For example, this indicates elements $v$ [2][0:12] of the array in device $(0)$ are transferred to an upper sleeve area of the array in device(1)

Lastly, other communication directives between accelerators are also proposed. Fig. 8 shows an example using the target gmove, the target bcast and the target reduction directives. In lines 3-4, 


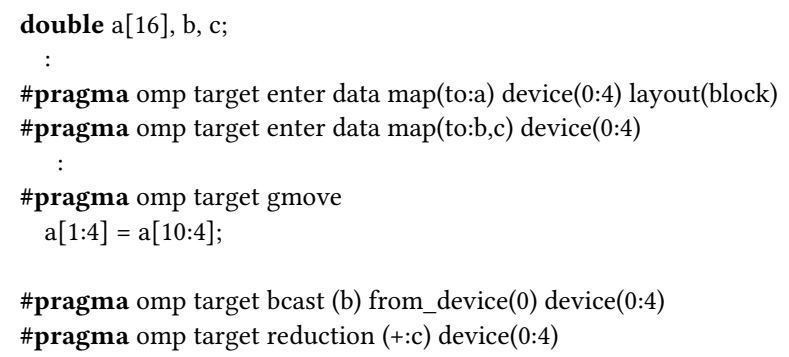

Figure 8: Examples of target gmove, target bcast, and target reduction directives in extended OpenMP

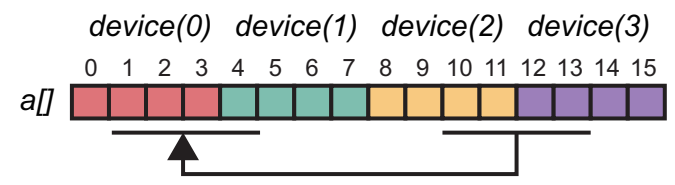

Figure 9: Concept of target gmove directive

the array $a[]$ which has 16 elements is divided on four accelerators. Scalar variables $b$ and $c$ are duplicated and stored in four accelerators redundantly because there is no layout clause. In line 6, the target gmove directive generates a communication for the distributed array in the form of the following assignment statement with array section. Fig. 9 shows a concept of the communication. The elements of $a[10: 4]$ on device (2:2) are copied to $a[1: 4]$ on device $(0: 2)$. The advantage of the target gmove directive can generate communication without being aware of how the distributed array is divided. This is a PGAS-like operation, and it is necessary for users to explicitly specify that communication will occur between the accelerators. In line 9, the target bcast directive executes broadcast communication from a specified accelerator. In this example, the scalar variable $b$ on device $(0)$ is broadcasted to the other accelerators. In line 10 , the target reduction directive executes the reduction operation on the scalar variable $c$. The total of the variable $c$ on all the accelerators is stored in the same variable $c$. The target reduction directive supports typical operators (such as "+," "*”, and "MAX”).

\subsection{Implementation}

To compile code including the OpenMP extension, we extend Omni Compiler[13,16] which is a source-to-source compiler infrastructure. Fig. 10 shows the compile flow of Omni Compiler. First of all, Omni Compiler translates the OpenMP extensions in a user code into general OpenMP directives and runtime calls. The runtime calls are developed using the general OpenMP APIs. Moreover, a part of the base language $(\mathrm{C} / \mathrm{C}++/$ Fortran $)$ are modified if necessary. Next, the translated code is compiled by a native compiler to generate an execution file with linking the runtime. Since the translated code uses only general OpenMP directives and the runtime calls, the execution file can execute on any accelerator. Note that some of the later works manually converts the user code because some functions of Omni Compiler is being developed.

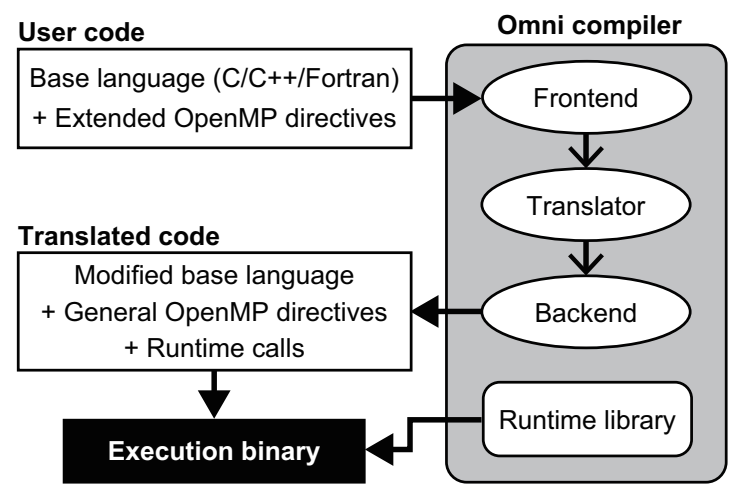

Figure 10: Compile flow in Omni Compiler

Fig. 11 shows a part of a translated code from Fig. 4 . Note that "_omni_" is added to the prefix of the variable name automatically generated by Omni Compiler so that user variable names are not wrapped. Also, a character string with all capital letters with "OMNI" as a prefix is a macro constant defined in Omni Compiler. Lines 2-4 of Fig. 11 correspond to line 2 of Fig. 4 . The function _omni_target_map_start(), which is provided by the runtime, is to allocate memory on the accelerator and associates it with a host address of an array. The argument of the function is the type of the map clause, a host address of an array, a size of the array, a presence or not of a sleeve area, and information of device set. The return value is a pointer array, and each element stores a top address of a memory allocated on each accelerator. In lines 6-16, the parallel directive spawns host threads as shown in Fig. 2. In lines 9-11, the top address of the memory on the accelerator in charge of each thread is stored in a temporary pointer. In line 12 , the function_omni_calc_iterations() calculates the number of iterations of the following loop statement for each thread. In line 13, the is_device_ptr clause is used to indicate that a list item is a device pointer. In lines 14-15, each thread processes its own responsible range. In lines 17-19, the function_omni_target_map_end() releases the memory on the accelerator and transfers the result from an accelerator to a host if necessary. Note that communication directives such as target reflect described in Section 4.2 are replaced with communication functions provided by the runtime one-to-one correspondence.

\section{EVALUATION}

\subsection{Overview}

This section evaluates the performance of the STREAM Triad and the HIMENO benchmarks[5]. The HIMENO benchmark evaluates the performance of incompressible fluid analysis code in Flops. The HIMENO benchmark is a typical stencil application, which is similar to the Laplace equation shown in Fig. 5.

Table 1 shows our computing environment for the evaluation. The compute node has two NVIDIA Tesla K80 each of which consists of two GK210 processors. That is, it can be used as a total of four GPUs. As a native compiler for Omni Compiler, we use the latest Clang-ykt compiler[3]. For reference, we measure the performance not only GPUs but also a single CPU socket with 16 threads. 


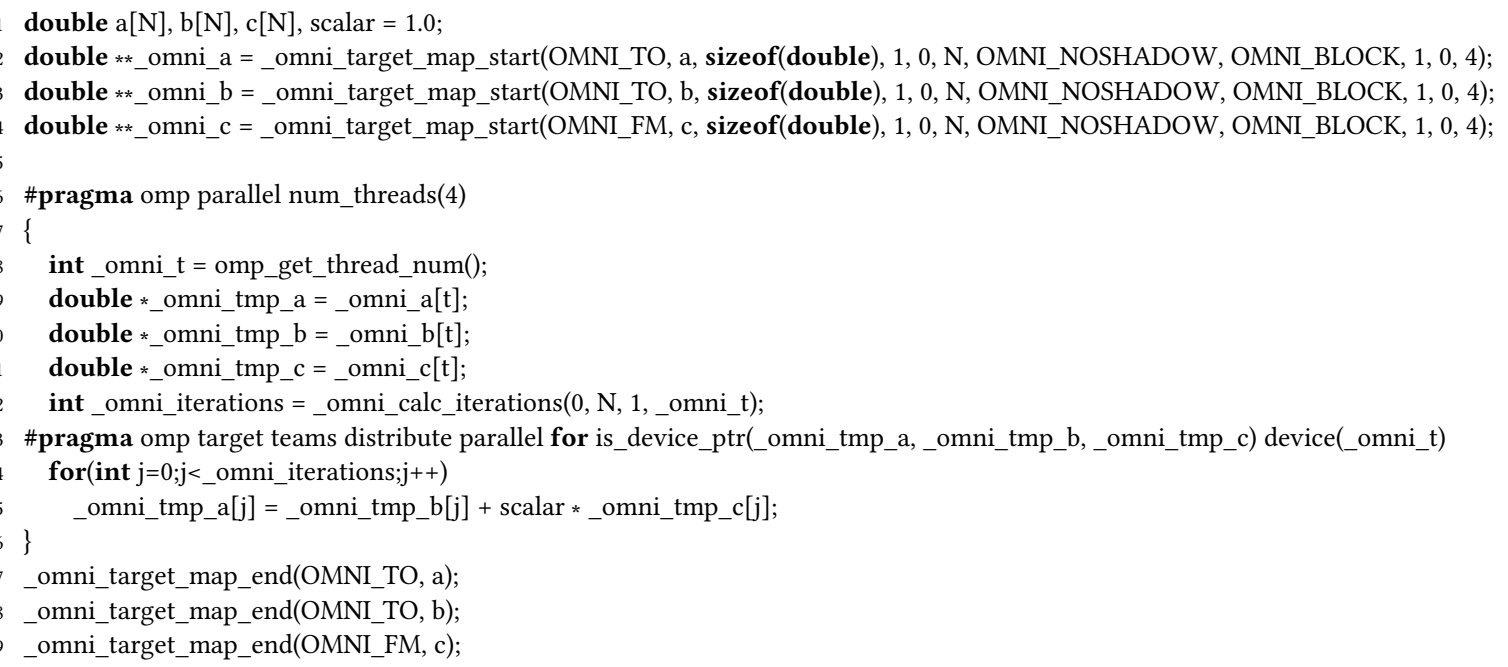

Figure 11: Translated code for multiple accelerators from Fig. 4

Table 1: Specification of compute node

\begin{tabular}{l|l}
\hline \hline CPU & Intel Xeon E5-2698v3 2.3GHz (16 Cores) x 2 Sockets \\
Memory & DDR3-2133, 128GB, 68 GB/s \\
GPU & NVIDIA Tesla K80 (GK210 x 2) x 2 GPUs, \\
Software & GDDR5 12GB, 240 GB/s x 2 \\
\hline
\end{tabular}

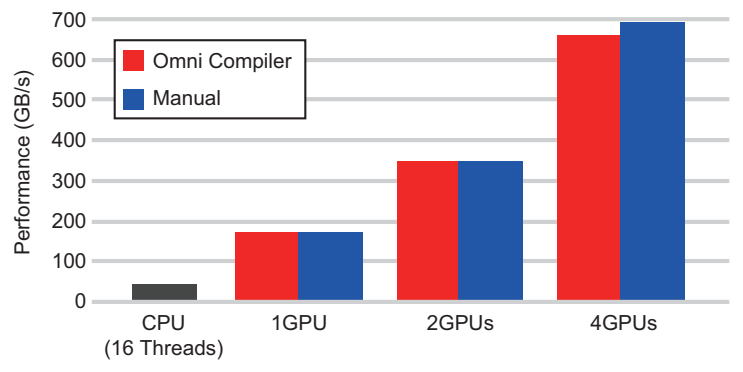

Figure 12: Performance results of STREAM Triad

The compile option for CPU is -O3 -fopenmp whereas that for GPU is -O3 -fopenmp -fopenmp-targets=nvptx64-nvidia-cuda.

\subsection{Performance Results}

5.2.1 STREAM Triad. Fig. 12 shows the performance results of the STREAM Triad benchmark. The item "Omni Compiler" means the results using the code shown in Fig. 4 with Omni Compiler. For comparison purpose, the item "Manual" there means the results using the code shown in Fig. 2, which is manually parallelized to use multiple accelerators. The result of "2 GPUs" uses two GPUs in a single K80 GPU. The size of array is $2^{28}(N=268,435,456)$.

Fig. 12 indicates that the results using GPU are better than the result using CPU. The results of "Omni Compiler" and "Manual" are almost the same, but only for 4 GPUs, the results of "Manual" are a little better. The reason is that the code translated by Omni Compiler is a little more difficult than the code manually parallelized, so it seems that optimization of the native compiler does not work a little.

5.2.2 HIMENO benchmark. Fig. 13 shows a part of the HIMENO benchmark code using extended OpenMP. The code is very similar to the code of the Laplace equation shown in Fig. 5. In lines 9-11, the target enter data directives with device and layout clauses declare distributed arrays. The distributed arrays are divided in the MIMAX dimension, and a sleeve area added to the dimension of the array $p[][][]$. In lines 15 and 25, the target teams distribute parallel for directives with the layout clause executes the following loop statements in multiple accelerators. In line 15, the collapse clause is the same means as the original OpenMP clause, whereas the reduction clause is to perform reduction operation among all accelerators because of a layout clause.

Fig. 14 shows the performance results of the HIMENO benchmark where the problem size is (MIMAX, MJMAX, MKMAX) = $(256,256,512)$. This indicates that the results using GPU are better than the result using CPU, and that the performance of the "Omni Compiler" is almost the same as those of "Manual."

\subsection{Discussion}

As for the performance, from the results of Fig. 12 and Fig. 14, the proposed OpenMP extension achieves almost the same performance as the manually generated program. As for the productivity, both the STREAM Triad and the Himeno benchmarks are implemented by only adding some clauses and directives to a single accelerator version of their codes.

Comparing with existing researches described in Section 2, it can be said that the proposed OpenMP extension can do programming more easily than [28] because the proposed directives can divide data and tasks. Also, unlike [23], the proposed directives can upload data at an arbitrary position. Moreover, unlike [12], the 


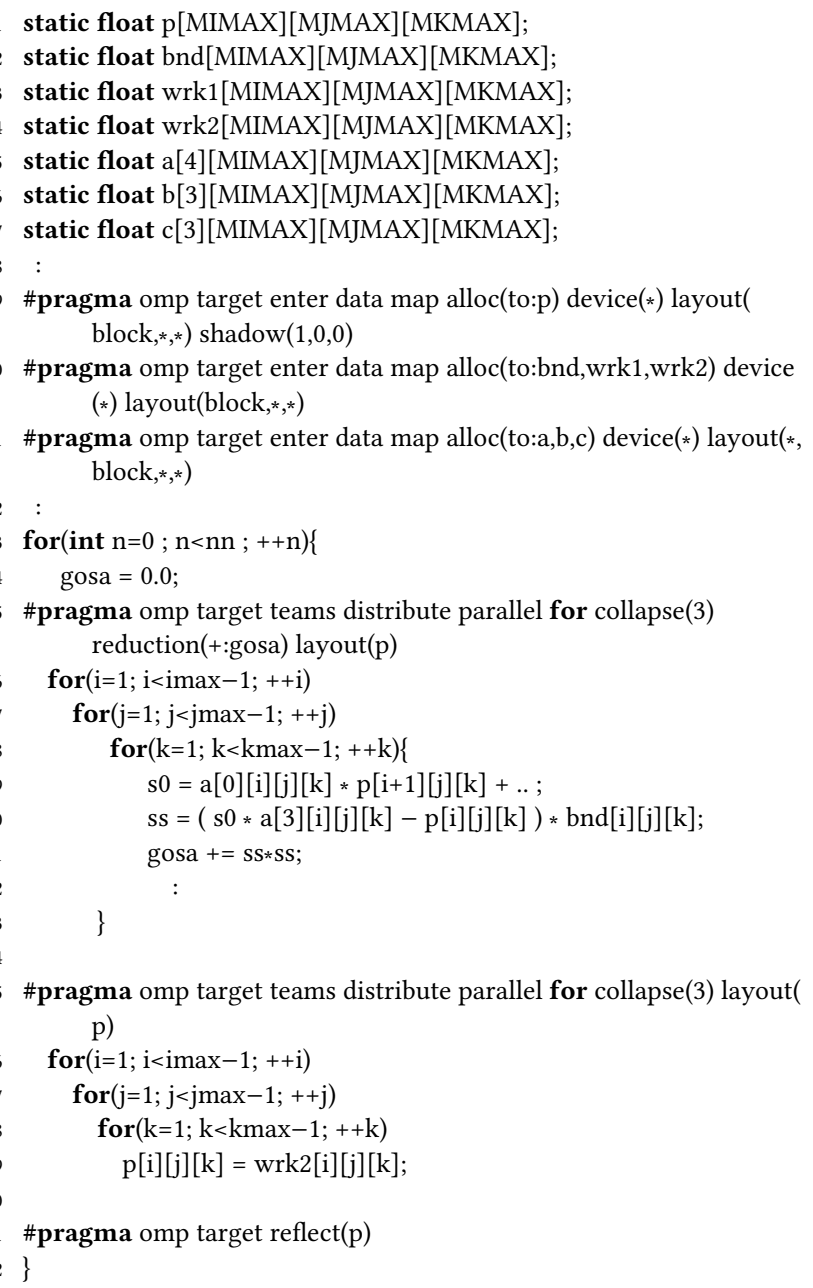

Figure 13: A part of HIMENO benchmark using extended OpenMP

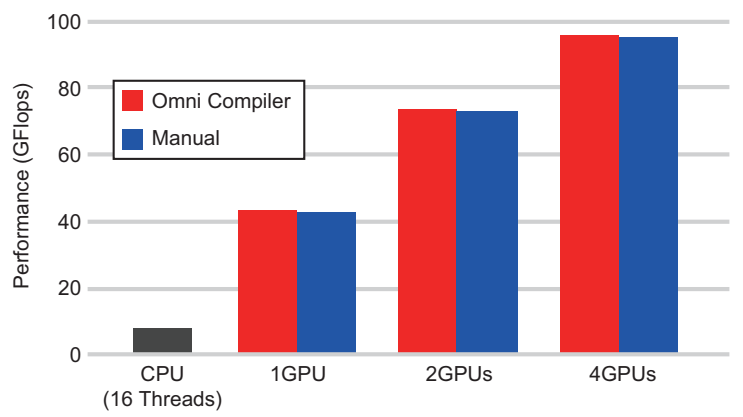

Figure 14: Performance results of HIMENO benchmark

proposed directives can utilize all accelerator memory effectively. In addition, while [23] and [12] may insert communication implicitly, our proposed OpenMP extension makes users insert communication explicitly using simple directives to perform performance tuning easily. Note that our idea may be inferior in terms of productivity compared to [12]. However, the purpose of [12] is to execute a code on multiple accelerators environment without changing code on a single accelerator environment, so the purpose is different from ours. In addition, unlike [10, 26], our method does not change the host side programming.

\section{CONCLUSION AND FUTURE RESEARCH}

In this paper, we propose the OpenMP extension that can divide data and tasks to use multiple accelerators in ease. We implement the STREAM Triad and the HIMENO benchmarks using the OpenMP extension. As a result of measuring the performance on a compute node with up to four GPUs, we confirm that the proposed OpenMP extension demonstrates sufficient performance.

Future research includes creating a specification for the OpenMP extension and developing other benchmarks with using collective communication directives described in Fig. 8. Moreover, to deal with accelerated clusters, we also consider combining the OpenMP extension with XMP.

\section{ACKNOWLEDGEMENT}

This work was supported by JSPS KAKENHI Grant Number 18K11331.

\section{REFERENCES}

[1] Chamberlain B.L. and Callahan D. and Zima H.P. 2007. Parallel Programmability and the Chapel Language. Int. F. High Perform. Comput. Appl. 21, 3 (Aug. 2007) 291-312.

[2] Charles Philippe and Grothoff Christian and Saraswat Vijay and Donawa Christopher and Kielstra Allan and Ebcioglu Kemal and von Praun Christoph and Sarkar Vivek. 2005. X10: An Object-oriented Approach to Non-uniform Cluster Computing. SIGPLAN Not. 40, 10 (Oct. 2005), 519-538.

[3] clang-ytk. 2018. clang-ykt/clang. (2018). https://github.com/clang-ykt/clang/.

[4] Free Software Foundation, Inc. 2017. Offloading Support in GCC. (2017) https://gcc.gnu.org/wiki/Offloading.

[5] Ryutaro Himeno. 2001. Himeno benchmark.

(2001) http://accc.riken.jp/en/supercom/himenobmt/

[6] J. Guan and S. Yan and J. M. Jin. 2013. An OpenMP-CUDA Implementation of Multilevel Fast Multipole Algorithm for Electromagnetic Simulation on MultiGPU Computing Systems. IEEE Transactions on Antennas and Propagation 61, 7 (July 2013), 3607-3616. https://doi.org/10.1109/TAP.2013.2258882

[7] Jack Dongarra and Piotr Luszczek. 2018. HPC Challenge Benchmarks. (2018). http://icl.cs.utk.edu/hpcc/.

[8] James Beyer and Bronis R. de Supinski. 2016. IWOMP 2016 Tutorial: OpenMP Accelerator Model. (2016) http://iwomp2016.riken.jp/wp-content/uploads/2016/10/tutorial-accelerator.pdf.

[9] Liao, Chunhua and Yan, Yonghong and de Supinski, Bronis R. and Quinlan, Daniel J. and Chapman, Barbara. 2013. Early Experiences with the OpenMP Accelerator Model. In OpenMP in the Era of Low Power Devices and Accelerators. Springer Berlin Heidelberg, Berlin, Heidelberg, 84-98.

[10] Masahiro Nakao and Hitoshi Murai and Hidetoshi Iwashita and Akihiro Tabuchi and Taisuke Boku and Mitsuhisa Sato. 2017. Implementing Lattice QCD Application with XcalableACC Language on Accelerated Cluster. In 2017 IEEE International Conference on Cluster Computing (CLUSTER). 429-438. https://doi.org/10.1109/CLUSTER.2017.58

[11] Masahiro Nakao and Hitoshi Murai and Hidetoshi Iwashita and Taisuke Boku and Mitsuhisa Sato. 2017. Implementation and evaluation of the HPC challenge benchmark in the XcalableMP PGAS language. The Inter national Journal of High Performance Computing Applications (2017), 1-14 https://doi.org/10.1177/1094342017698214

[12] Matsumura, Kazuaki and Sato, Mitsuhisa and Boku, Taisuke and Podobas, Artur and Matsuoka, Satoshi. 2018. MACC: An OpenACC Transpiler for Automatic Multi-GPU Use. In Supercomputing Frontiers. Springer International Publishing, Cham, 109-127.

[13] Mitsuhisa Sato, Hitoshi Murai, Masahiro Nakao, Hidetoshi Iwashita, Jinpil Lee, Akihiro Tabuchi. 2016. Omni Compiler and XcodeML An Infrastructure for Source-to-Source Transformation. Platform for Advanced Scientific Computing Conference (PASC16). (2016) http://omni-compiler.org/download/papers/PASC2016.pdf 
[14] NVIDIA Corporation. 2018. https://developer.nvidia.com/cuda-toolkit.

[15] Oak Ridge Leadership Computing Facility. 2018. Introducing Summit. (2018). https://www.olcf.ornl.gov/summit/.

[16] Omni Compiler Project. 2018. Omni Compiler. (2018). http://omni-compiler.org.

[17] OpenACC organization. $2018 . \quad$ OpenACC. (2018). http: //www. openacc-standard.org.

[18] OpenMP Architecture Review Board. 2018. OpenMP. (2018). https: //www. openmp.org.

[19] OpenMP Architecture Review Board. 2018. OpenMP Compilers and Tools (2018). https://www.openmp.org/resources/openmp-compilers-tools/.

[20] R. Numwich and J. Reid. 1998. Co-Array Fortran for parallel programming. Technical Report RAL-TR-1998-060. Rutherford Appleton Laboratory.

[21] S. Potluri and H. Wang and D. Bureddy and A. K. Singh and C. Rosales and D. K. Panda. 2012. Optimizing MPI Communication on Multi-GPU Systems Using CUDA Inter-Process Communication. In 2012 IEEE 26th International Parallel and Distributed Processing Symposium Workshops PhD Forum. 1848-1857. https://doi.org/10.1109/IPDPSW.2012.228

[22] T. El-Ghazawi and F. Cantonnet. 2002. UPC Performance and Potential: A NPB Experimental Study. In Proceedings of the 2002 ACM/IEEE Conference on Supercomputing. 1-26.

[23] T. Komoda and S. Miwa and H. Nakamura and N. Maruyama. 2013. Integrating Multi-GPU Execution in an OpenACC Compiler. In 2013 42nd International Conference on Parallel Processing. 260-269. https://doi.org/10.1109/ICPP.2013.35

[24] The Khronos Group Inc. 2018. OpenCL : The Open Standard for Parallel Programming of Heterogeneous Systems. (2018). https://www.khronos.org/opencl/.

[25] Michael Wolfe. 2014. SCALING OPENACC APPLICATIONS ACROSS MULTIPLE GPUS. (2014). http://on-demand.gputechconf.com/gtc/2014/presentations/ S4474-scaling-openacc-across-multiple-gpus.pdf.

[26] XcalableMP Specification Working Group. 2018. XcalableACC Specification. (2018). http://xcalablemp.org/XACC.html.

[27] XcalableMP Specification Working Group. 2018. XcalableMP Specification. (2018). http://xcalablemp.org/specification.

[28] Xu, Rengan and Tian, Xiaonan and Chandrasekaran, Sunita and Chapman, Barbara. 2016. Multi-GPU Support on Single Node Using Directive-based Programming Model. Sci. Program. 2015, Article 3 (Jan. 2016), 1 pages. https://doi.org/10.1155/2015/621730 